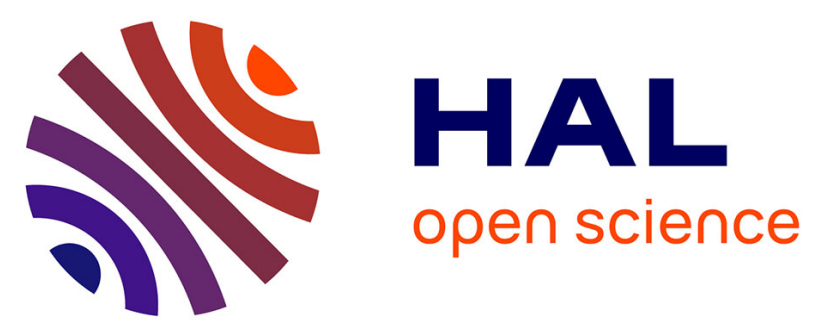

\title{
Near Unity Absorption in Nanocrystal Based Short Wave Infrared Photodetectors using Guided Mode Resonators
}

\author{
Audrey Chu, Charlie Gréboval, Nicolas Goubet, Bertille Martinez, Clément \\ Livache, Junling Qu, Prachi Rastogi, Francesco Andrea Bresciani, Yoann \\ Prado, Stéphan Suffit, et al.
}

\section{To cite this version:}

Audrey Chu, Charlie Gréboval, Nicolas Goubet, Bertille Martinez, Clément Livache, et al.. Near Unity Absorption in Nanocrystal Based Short Wave Infrared Photodetectors using Guided Mode Resonators. ACS photonics, 2019, 10.1021/acsphotonics.9b01015 . hal-02281355

\section{HAL Id: hal-02281355 \\ https://hal.science/hal-02281355}

Submitted on 30 Oct 2019

HAL is a multi-disciplinary open access archive for the deposit and dissemination of scientific research documents, whether they are published or not. The documents may come from teaching and research institutions in France or abroad, or from public or private research centers.
L'archive ouverte pluridisciplinaire HAL, est destinée au dépôt et à la diffusion de documents scientifiques de niveau recherche, publiés ou non, émanant des établissements d'enseignement et de recherche français ou étrangers, des laboratoires publics ou privés. 


\title{
Near Unity Absorption in Nanocrystal Based Short Wave Infrared Photodetectors using Guided Mode Resonators
}

\author{
Audrey Chu ${ }^{1,2}$, Charlie Gréboval ${ }^{1}$, Nicolas Goubet ${ }^{1,3}$, Bertille Martinez ${ }^{1,5}$, Clément \\ Livache $^{1,5}$, Junling $\mathrm{Qu}^{1}$, Prachi Rastogi ${ }^{1}$, Francesco Andrea Bresciani ${ }^{1}$, Yoann \\ Prado $^{1}$, Stephan Suffit ${ }^{4}$, Sandrine Ithurria ${ }^{5}$, Grégory Vincent ${ }^{2 *}$, Emmanuel Lhuillier ${ }^{1 *}$ \\ ${ }^{1}$ Sorbonne Université, CNRS, Institut des NanoSciences de Paris, INSP, F-75005 \\ Paris, France \\ 2 ONERA - The French Aerospace Lab, 6, chemin de la Vauve aux Granges, BP \\ 80100, F-91123 Palaiseau, France \\ ${ }^{3}$ Sorbonne Université, CNRS, De la Molécule aux Nano-objets: Réactivité, \\ Interactions et Spectroscopies, MONARIS, F-75005 Paris, France \\ ${ }^{4}$ Laboratoire Matériaux et Phénomènes Quantiques, UMR 7162 CNRS, Université \\ Paris Diderot, Paris, France \\ ${ }^{5}$ Laboratoire de Physique et d'Etude des Matériaux, ESPCI-Paris, PSL Research \\ University, Sorbonne Université UPMC Univ Paris 06, CNRS, 10 rue Vauquelin \\ 75005 Paris, France.
}

\begin{abstract}
Nanocrystals appear as versatile building blocks for the design of low-cost optoelectronic devices. The design of infrared sensors based on nanocrystals is currently facing a key limitation: the short carrier diffusion length resulting from hopping transport makes that only a limited part of the incident light is absorbed. In order to enhance the device absorption, we use Guided Mode Resonance (GMR). The method appears to be quite versatile and is applied to both $\mathrm{PbS}$ and $\mathrm{HgTe}$ nanocrystals presenting respectively cut-off wavelengths at 1.7 and $2.6 \mu \mathrm{m}$. The designed electrodes present a large enhancement of the material responsivity around a factor of $\approx 250$, reaching external quantum efficiency of $86 \%$ for $\mathrm{PbS}$ and $340 \%$ for $\mathrm{HgTe}$. This increase of the response can be deconvoluted in a factor of 3 for the enhancement of the absorption and a factor of 80 for the photocurrent gain. The method can also be suited to finely tune the cut-off wavelength of the material thanks to geometrical parameters at the device level. The obtained devices are now only limited by the material noise.
\end{abstract}

Keywords: nanocrystals, short wave infrared, infrared detection, plasmonic resonance, $\mathrm{PbS}, \mathrm{HgTe}$, guided mode resonance.

To whom correspondence should be sent: el@insp.upmc.fr and gregory.vincent@onera.fr 
Colloidal Quantum Dots (CQDs) have raised a significant interest thanks to their widely tunable optical features from the UV to the $\mathrm{THz}^{1,2}$ ranges. Their integration into optoelectronic devices raises another level of challenge because of the combination of optical properties and electronic transport. A key step in this direction relates to the development of ligand exchange procedures which allow to achieve stronger electronic coupling as well as tuning the CQD doping ${ }^{3-6}$. Thanks to ligand exchange, the mobility, which is a macroscopic measurement of the local CQD coupling, has been increased from $10^{-6} \mathrm{~cm}^{2} \cdot V^{-1} \cdot \mathrm{s}^{-1}$ for CQDs capped with long ligands to $10^{-3}-10^{-2} \mathrm{~cm}^{2} \cdot \mathrm{V}^{-1} \cdot \mathrm{s}^{-1}$ with short organic ligands and even above one with ionic/inorganic surface chemistry ${ }^{7-11}$. However, in spite of these progresses, the carrier diffusion length remains limited typically below $100 \mathrm{~nm}$, which is, at least, one order of magnitude smaller than the absorption depth ${ }^{12,13}$. As a result, the design of CQD-based photodetectors results from a trade-off between the absorption and the charge conduction. Due to this short diffusion length, the thickness of the CQD-based absorbing layer remains below 500 $\mathrm{nm}$ which makes that only 10 to $50 \%$ of the incident light is absorbed and the amount of photocarriers that reach the electrodes is even lower. This is a major challenge for the design of CQD-based photodetector that needs to be tackled.

In the recent years, large progresses have been obtained for the design of short-wave infrared detectors ${ }^{14,15}$ using $\mathrm{PbS}^{16-20}$ and $\mathrm{HgTe}^{21-26} \mathrm{CQDs}$, as a possible low-cost alternative to InGaAs technology. A possible strategy to overcome the low absorption in CQD-based light detectors, due to the limited thickness of the CQD layer, is to concentrate the electromagnetic field compared to its plane wave propagation. This is in particular possible with the introduction of plasmonic resonances ${ }^{27}$. While this field has been widely investigated for conventional semiconductor solar cells ${ }^{28,29}$ and infrared sensors ${ }^{30-33}$, far less works have been done on this question for CQD-based devices ${ }^{34-43}$.

In this paper, we focus on the short-wave infrared (SWIR, $\lambda<1.7 \mu \mathrm{m})$ and extended short-wave infrared (E-SWIR, $\lambda<2.5 \mu \mathrm{m})$ ranges using respectively $\mathrm{PbS}$ and $\mathrm{HgTe}$ CQDs as active material. We propose to implement Guided Mode Resonance $(\mathrm{GMR})^{44}$ as a strategy to achieve absorption close to unity while using thin films $(200-300 \mathrm{~nm})$ and to improve photocarrier collection. We demonstrate that not only we obtain good qualitative and quantitative agreement with electromagnetic simulation, but also that GMR is a viable path to boost device performance and tune its spectral response. 


\section{Discussion}

We start by synthetizing $\mathrm{PbS}^{12}$ and $\mathrm{HgTe}^{45}$ nanocrystals with excitonic feature respectively at $1.55 \mu \mathrm{m}\left(0.8 \mathrm{eV}\right.$ or $6000 \mathrm{~cm}^{-1}$, later called PbS 6k) and $2.6 \mu \mathrm{m}(\approx 0.5 \mathrm{eV}$ or $4000 \mathrm{~cm}^{-1}$, called $\mathrm{HgTe} 4 \mathrm{k}$ ) using established methods from the literature, see spectra in Figure 1a and Transmission Electron Microscopy (TEM) image in Figure 1b and c. Both materials can be deposited onto interdigitated electrodes with $30-\mu \mathrm{m}$ period to build a conductive device. In this case, in order to build thick films (typically $200 \mathrm{~nm}$ ), a multi-layer deposition is required. For each layer, we perform a ligand exchange step from long alkyl chains, oleic acid and dodecanethiol for respectively $\mathrm{PbS}$ and $\mathrm{HgTe}$, towards ethanedithiol to ensure a strong interparticle coupling. Both materials present a photoresponse as shown by the modulation of the I-V curve under illumination, see Figure $1 \mathrm{~d}$ and e. For such films, the responsivity has been estimated to be around $1 \mathrm{~mA} \cdot \mathrm{W}^{-1}$ for the two materials, under $1 \mathrm{~V}$ of applied bias and for room temperature operation, see Figure S1 and S2. Such low responsivity is the result of $(I)$ a weak absorption $\approx 12 \%$ considering the coefficient of absorption $\alpha \approx 3 \times 10^{3} \mathrm{~cm}^{-1}$ 13,46 for $\mathrm{HgTe}$ and a film thickness of $200 \mathrm{~nm}$ and (ii) a poor charge collection due to the short diffusion length. These limitations come in addition with the presence of $1 / f$ noise ${ }^{47-49}$, see Figure $S 1$ and S2, to finally lead to low signal over noise ratio (i.e. detectivity) around $10^{7}$ Jones.
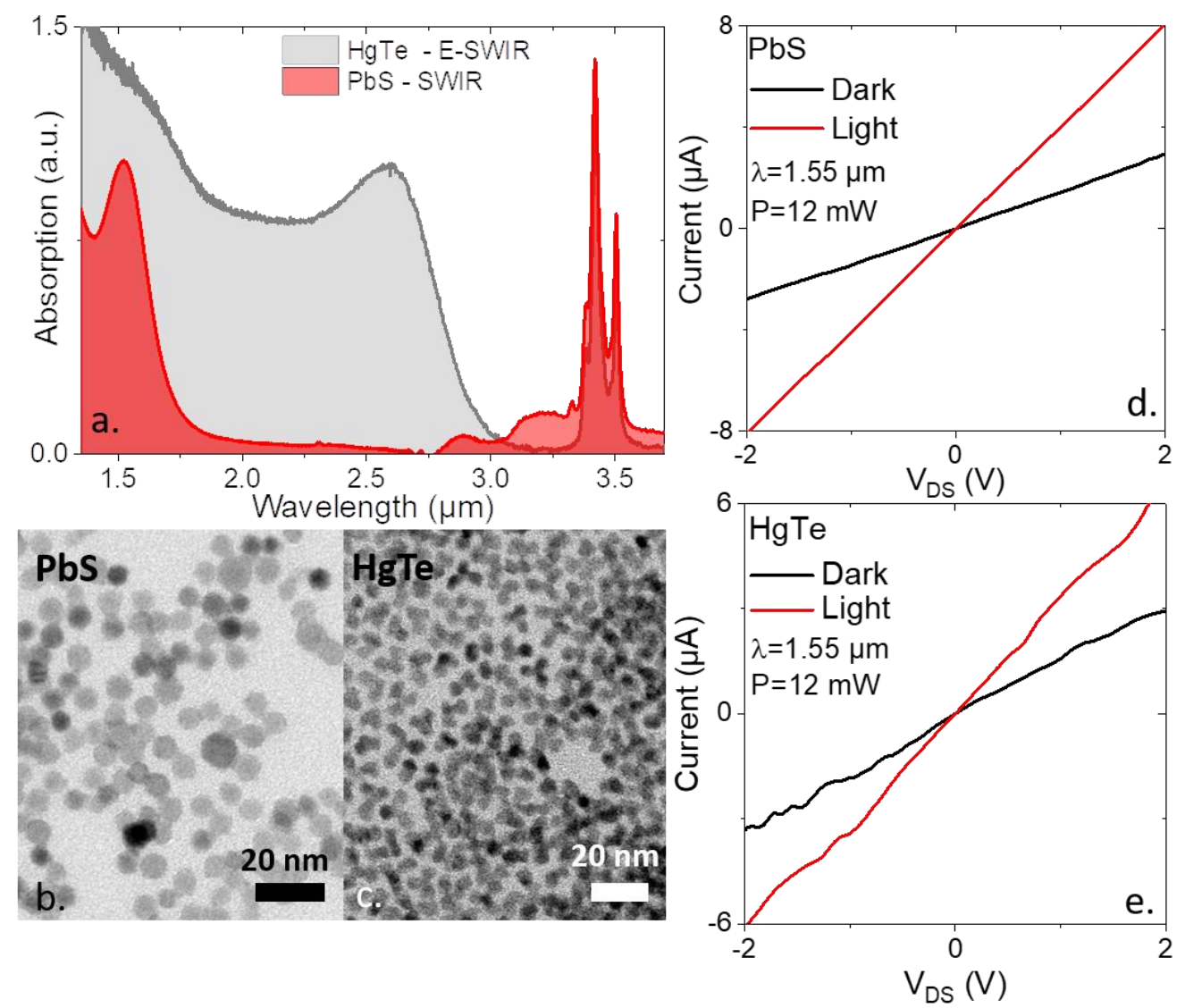

Figure 1 Material characterizations. a. Absorption spectra of PbS and $\mathrm{HgTe}$ nanocrystals used for this study. b. (resp. c.) Transmission electron microscopy images 
of PbS (resp. HgTe) nanocrystals. d. (resp. e.) I-V curves under dark condition and under illumination $(\lambda=1.55 \mu \mathrm{m}-\mathrm{P}=12 \mathrm{~mW}$ ) for $\mathrm{PbS}$ (resp. HgTe) nanocrystal 200-nm thick films measured at room temperature.

To address the difficulties resulting from both weak absorption and poor charge collection, we propose an architecture based on Guided-Mode Resonances (GMR). This structure not only allows the introduction of optical resonances to enhance the light-matter coupling but also relies on the shorter inter-electrodes spacing to reduce the transit time and thus achieve photoconductive gain ${ }^{50-52}$. Compared to the work done on non-colloidal semiconductors, the main challenge is to design a structure which accounts for the fragility of the colloidal materials. PbS nanocrystals can easily be oxidized, while $\mathrm{HgTe}$ nanocrystals sinter upon annealing even at relatively low temperatures ${ }^{53}$. Our approach is to design a structure in which all lithography steps are conducted before the CQD deposition, see Figure S3 for fabrication details.

The GMR structure consists in a thin film deposited onto a grating pattern. From an electromagnetic point of view, the grating, via diffracted waves, aims to couple incoming light within a guided-mode of the thin film. Depending on the materials and geometries, this coupling gives rise to resonant transmission, reflection or absorption of light, at a particular wavelength $\lambda_{R}$. Such GMR architecture has been widely used for band-pass filtering ${ }^{54}$ or notch filtering ${ }^{55}$, and more rarely for resonant absorption ${ }^{31}$. Its main advantage, particularly compared to Fabry-Perot resonators, stands in the fact that the resonant wavelength is tunable with the period of the grating (i.e. through a geometrical factor rather than by tuning a material parameter). Thus, multispectral patterns can be achieved on a sample with a single lithography step. ${ }^{56}$ In our study, grating is made of gold and the waveguide is the CQD film.

Figure 2 provides a schematic of the three structures we studied: (i) the conventional interdigitated electrodes with a $30 \mu \mathrm{m}$ period (Figure $2 \mathrm{a}, \mathrm{d} \& \mathrm{~g}$ ), (ii) the same electrode pattern with a back side-mirror (Figure $2 b, e \& h$ ) and the micro-electrodes used to induce GMR (Figure 2c, $f \&$ i). The absorption of the conventional interdigitated electrodes corresponds mainly to one passing of the incident light through the CQD film (Figure 2g). The addition of the gold mirror allows reflection of the light and so, a second passing of the incident light through the film. Note that it is similar to the optical path in vertical geometry devices where metallic back electrode can also reflect the incident light towards the absorbing CQD film (Figure 2h). Last, Figure 2f illustrates light trapping in the CQD film thanks to diffracted orders. For sake of comparison, we calculate the predicted spectrum for the three types of electrodes. These calculations have been achieved with Reticolo ${ }^{\circledR}$ software ${ }^{57}$ based on RCWA (Rigorous CoupledWave Analysis). Compared to conventional electrodes, the metallic mirror leads to a $45 \%$ increase of the absorption (Figure $2 \mathrm{j}$ and $\mathrm{k}$ ). This is less than a factor of two due to the already existing partial reflection of the light at the $\mathrm{CQD} / \mathrm{SiO}_{2}$ interface (Figure 2 g). With the GMR electrodes, the absorption reaches $70 \%$ (Figure 2I) of the total 
incident light, which is 2.9 times more than the absorption obtained for conventional electrodes (Figure 2j and I). We will now explain the resonant mechanisms involved in GMR and the basic design rules.
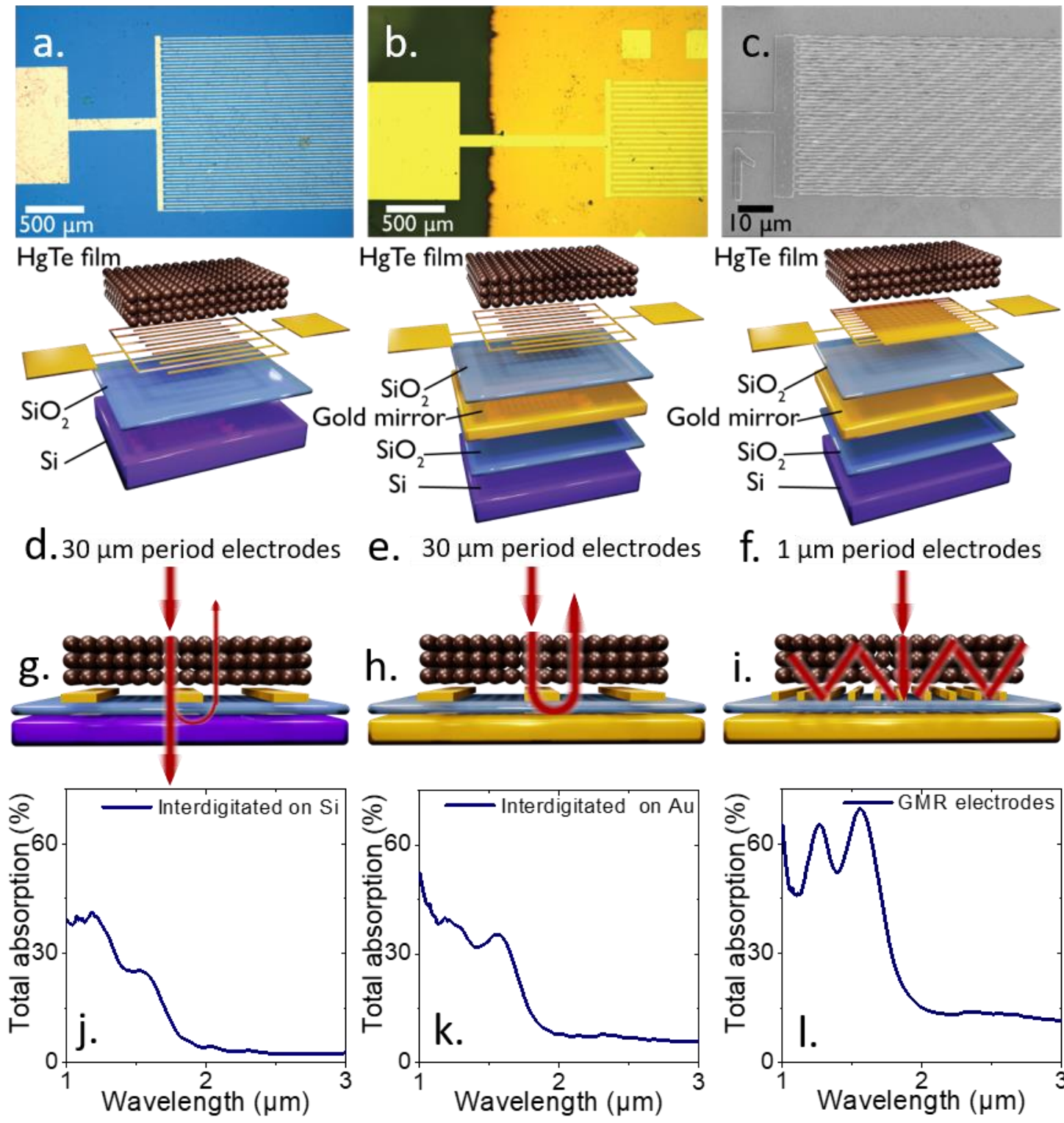

e. $30 \mu \mathrm{m}$ period electrodes

f. $1 \mu \mathrm{m}$ period electrodes
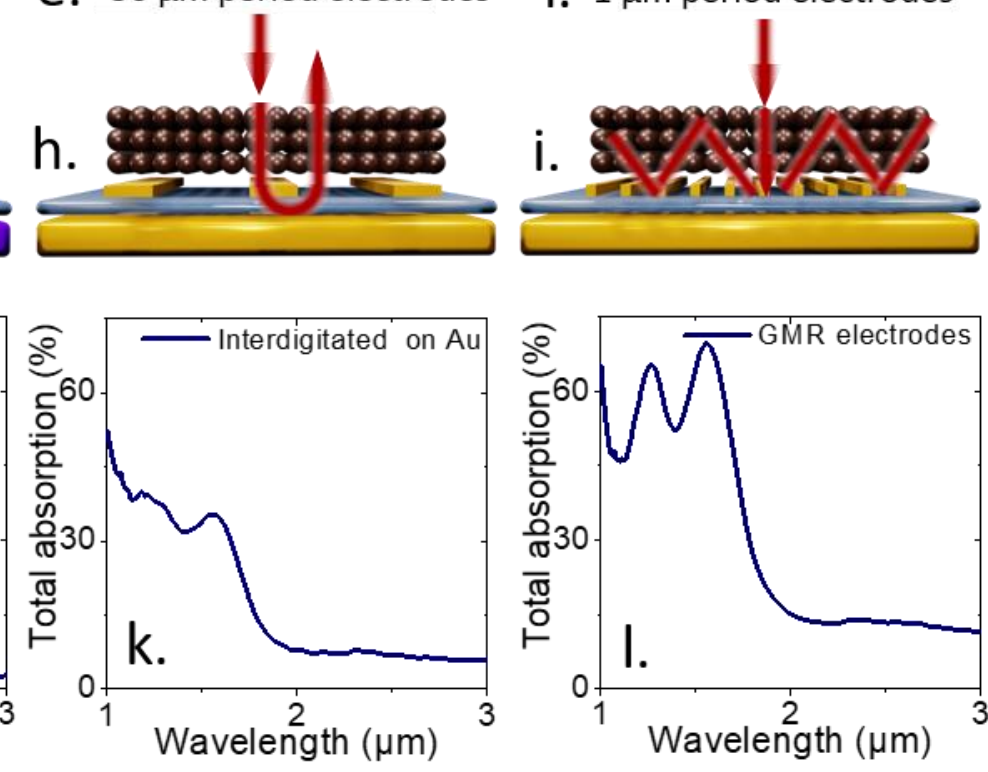

Figure 2: Description of used structures. a. Optical microscopy image of the conventional interdigitated gold electrodes deposited on a $\mathrm{Si} \mathrm{SiO}_{2}$ substrate. b. Optical microscopy image of the interdigitated electrodes made of gold contact deposited on a $\mathrm{Au} / \mathrm{SiO}_{2}$ substrate. c. Scanning electron microscopy image of the interdigitated gold electrodes on a $\mathrm{Au} / \mathrm{SiO}_{2}$ substrate designed to induce GMR. d-f. Schematics of respectively $d$. the conventional interdigitated gold electrodes deposited on a $\mathrm{Si}_{/} \mathrm{SiO}_{2}$ substrate, e. the interdigitated electrodes made of gold contact deposited on a $\mathrm{Au} / \mathrm{SiO}_{2}$ substrate and $f$. the interdigitated gold electrodes on a $\mathrm{Au} / \mathrm{SiO}_{2}$ substrate designed to induce GMR. g-i. Schematics of the cross section of $g$. the conventional interdigitated gold electrodes deposited on a $\mathrm{Si} \mathrm{SiO}_{2}$ substrate, $h$. the interdigitated electrodes made of gold contact deposited on a $\mathrm{Au} / \mathrm{SiO}_{2}$ substrate and $j$. the interdigitated gold electrodes on an $\mathrm{Au} / \mathrm{SiO}_{2}$ substrate designed to induce GMR. Red arrows correspond 
to light paths. $j-1$. Simulated absorption spectra for $j$. the conventional electrodes on $\mathrm{Si} / \mathrm{SiO}_{2}$, k. on $\mathrm{Au} / \mathrm{SiO}_{2}$ and for I. the electrodes on a $\mathrm{Au} / \mathrm{SiO}_{2}$ substrate designed to induce GMR. For figures $j, k$ and I the incident light is not polarized.

The proposed GMR structure is given in Figure 3b. The latter is designed to achieve a quasi-unity absorption for the TM mode (Transverse Magnetic - magnetic field parallel with the slits of the grating) of a 200-nm thick film of PbS CQDs at $\lambda_{R}=1.55 \mu \mathrm{m}$. The determination of the geometrical parameters starts with the period $p$ of the grating. It is set so that there is no diffracted orders reflected in air, but there must be diffracted orders in the CQD layer. Thus, the condition $p<\lambda_{R}<p . n_{C Q D}$ should be fulfilled under normal incidence ( $n_{C Q D}$ is the refractive index of the CQDs). Then, the period value is set in accordance with the CQD layer thickness $t_{C Q D}$, so that resonance occurs at the wavelength of interest. Finally, the thickness of the grating $t_{g}$ and the width of its openings $w$ are adjusted to maximize resonance. It is worth noting that only the grating is responsible for the resonant induce enhancement of the absorption (Figure S5). The back-side mirror is here to avoid loss of photon resulting from light transmission and induces a broad band enhancement of the light absorption.

Figure 3a shows calculated absorption spectra under normal incidence, for TM polarization (blue curve) and for transverse electric polarization (TE - electric field parallel with the slits - red curve) lights. The average of TM and TE curves is represented in black and corresponds to an unpolarized incident light. For a grating period of $1.0 \mu \mathrm{m}$ and a CQD film thickness of $t_{C Q D}=200 \mathrm{~nm}$, a single and almost total absorption peak ( $A_{t o t}^{T M}=99.3 \%$ ) occurs for $\lambda_{R}=1.55 \mu \mathrm{m}$ for TM polarization, due to GMR. For TE polarization, a shallow peak also occurs at $1.55 \mu \mathrm{m}$, which is due to $\mathrm{PbS}$ absorption and a stronger peak ( $A_{\text {tot }}^{T E}=80.4 \%$ ) occurs for $\lambda=1.25 \mu \mathrm{m}$, due to a second GMR.

The operation of the GMR electrodes (Figure 3b) is even better revealed by the map of electromagnetic absorption, see Figure $3 \mathrm{c}$ and $\mathrm{d}$. Refractive indices of involved materials and resultant geometrical parameters of the GMR resonator are given in Supplementary information, see Figure S4 and Table S1. The antinodes of the TM mode are mostly located on the top of the gold finger, while for TE mode they are localized between the fingers. This second resonance is quite interesting since it is far away from the metal and makes that $89 \%$ of the absorption is spatially located within the CQD (against $77 \%$ for the TM mode) The remaining absorption (11\%) occurs in the metal and leads to thermal losses and have consequently to be minimized. As a result, absorption in the CQDs are $A_{C Q D}^{T E}=A_{\text {tot }}^{T E} \times 0.89=71 \%$ and $A_{C Q D}^{T M}=A_{\text {tot }}^{T M} \times 0.77=$ $77 \%$ for TE and TM modes, respectively. 

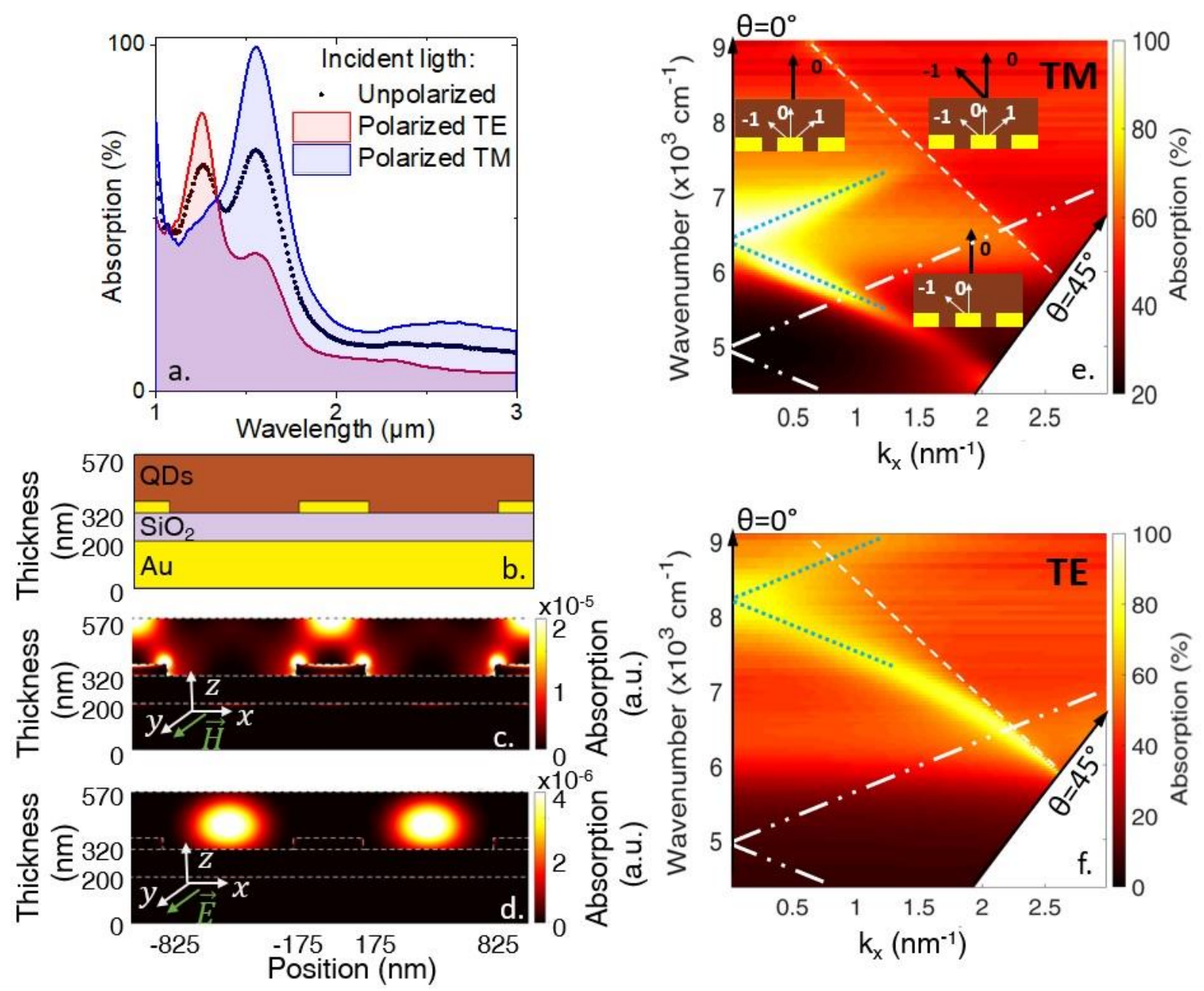

Figure 3 Electromagnetical properties of the GMR architecture. a. Simulated absorption spectra of the interdigitated electrodes on a gold/SiO $\mathrm{Substrate}_{2}$ designed to induce GMRs for an unpolarized (black), TM-polarized (blue) and TE-polarized (red) incident light. b. Cross section of the nanoelectrodes used to induce GMRs. c. Simulated cross section map of the absorption relative to the TM mode at $1.55 \mu \mathrm{m}$. $77 \%$ of the total absorption occurs in the CQDs. $d$. Simulated cross section map of the absorption relative to the TE mode at $1.25 \mu \mathrm{m}$. $89 \%$ of the total absorption occurs in the CQDs. All simulation are conducted using PbS 6k as absorbing material. e. (resp. f.) Angle-resolved specular TM (resp. TE) absorption simulation through interdigitated electrodes on a $\mathrm{Au} / \mathrm{SiO}_{2}$ substrate designed to induce GMRs, in linear color map as a function of $\sigma=1 / \lambda$, the wavenumber, and $k_{x}=2 \pi \cdot \sin (\theta) / \lambda$, the $x$ component of the incident wave vector. White dashed line corresponds to the appearance/disappearance of one diffracted order in the air. White dot-dashed lines correspond to the appearance/disappearance of one diffracted order in the CQD layer. Blue dotted lines correspond to the absorption peaks. Sketches represent the existing diffracted orders which propagate in the CQD array (brown box) and in air (top of the brown box).

The exact mechanisms of resonances can be deduced from angular dependency and absorption map. Figure $3 e$ and $f$ show calculated absorption diagram, given in linear 
color scale. We give on the $\mathrm{y}$-axis the wavenumber and on $\mathrm{x}$-axis $k_{x}$, which is the $\mathrm{x}$ component of the incoming wavevector. $k_{x}$ depends on incidence angle $\theta$ and on wavenumber $\sigma: k_{x}=2 \pi \cdot \sigma \cdot \sin (\theta)$. First of all, in the diagrams of Figure $3 e$ and $\mathrm{f}$, in white (dash or dash-dot) lines show the limits where first propagative orders are diffracted by the grating (in air or in CQDs, respectively). As expected, we thus see that resonance occurs when \pm 1 orders are diffracted in CQD only. The wave vector of the guided mode in the CQD is written as $\beta=n_{\text {eff }} .2 \pi / \lambda$, where $n_{\text {eff }}$ is the effective index of the mode. For GMR, this mode couples with the first diffracted orders $k_{\bar{x}}^{ \pm 1}$, and the resonance condition can thus be expressed as $k_{\bar{x}}^{ \pm 1}=2 \pi \cdot \sigma \cdot \sin (\theta) \pm$ $2 \pi / p \approx \beta=n_{e f f} .2 \pi$. $\sigma$. At $\theta=0^{\circ}$, we find $n_{\text {eff }}=\lambda / p=1.55$. This dispersion relation is plotted in Figure $3 e$ for $k_{x}^{ \pm 1}$, and we see the superimposition with the resonant absorption, which demonstrates the GMR mechanism. In this diagram, we also highlight an absorption peak at $\sigma=6250 \mathrm{~cm}^{-1}$, who has no angular dependency. This absorption is due to intrinsic absorption of $\mathrm{PbS}$ at this wavelength (see Figure 1a).

We have tested the robustness of this design toward changes of geometrical parameters. An increase of the film thickness leads to a redshift of the transition, see Figure S6. This nevertheless remains quite limited since a change by $50 \mathrm{~nm}$ of the thickness only shifts the resonance peak by its linewidth. We have also estimated how the spectrum is affected by the film surface topology, see Figures S8 and S9. Qualitatively, the conclusion remains unchanged and only a small shift (smaller than the peak linewidth) can be observed. Finally, angular dependence of the structure is discussed in Figure S10.

A comparison between the simulated spectra and the measured photocurrent is given in Figure 4. Several periods close to the optimal values have been designed to balance possible spectral shift resulting from a change of the CQD film thickness. The first striking observation is that the spectral response is drastically affected by a change in the period compared to the one obtained with non GMR electrodes, see Figure S1. We observe a large increase of the absorption around $1.55 \mu \mathrm{m}$, whose exact energy depends on the electrode period. The larger the period, the redder the resonance, see Figure $4 \mathrm{a}$ and $\mathrm{b}$. While the conventional interdigitated electrodes display no polarization effect (Figure S1b), the GMR electrodes present a clear polarization effect (Figure 4d), in good agreement with the estimated spectra, see Figure 4c. 

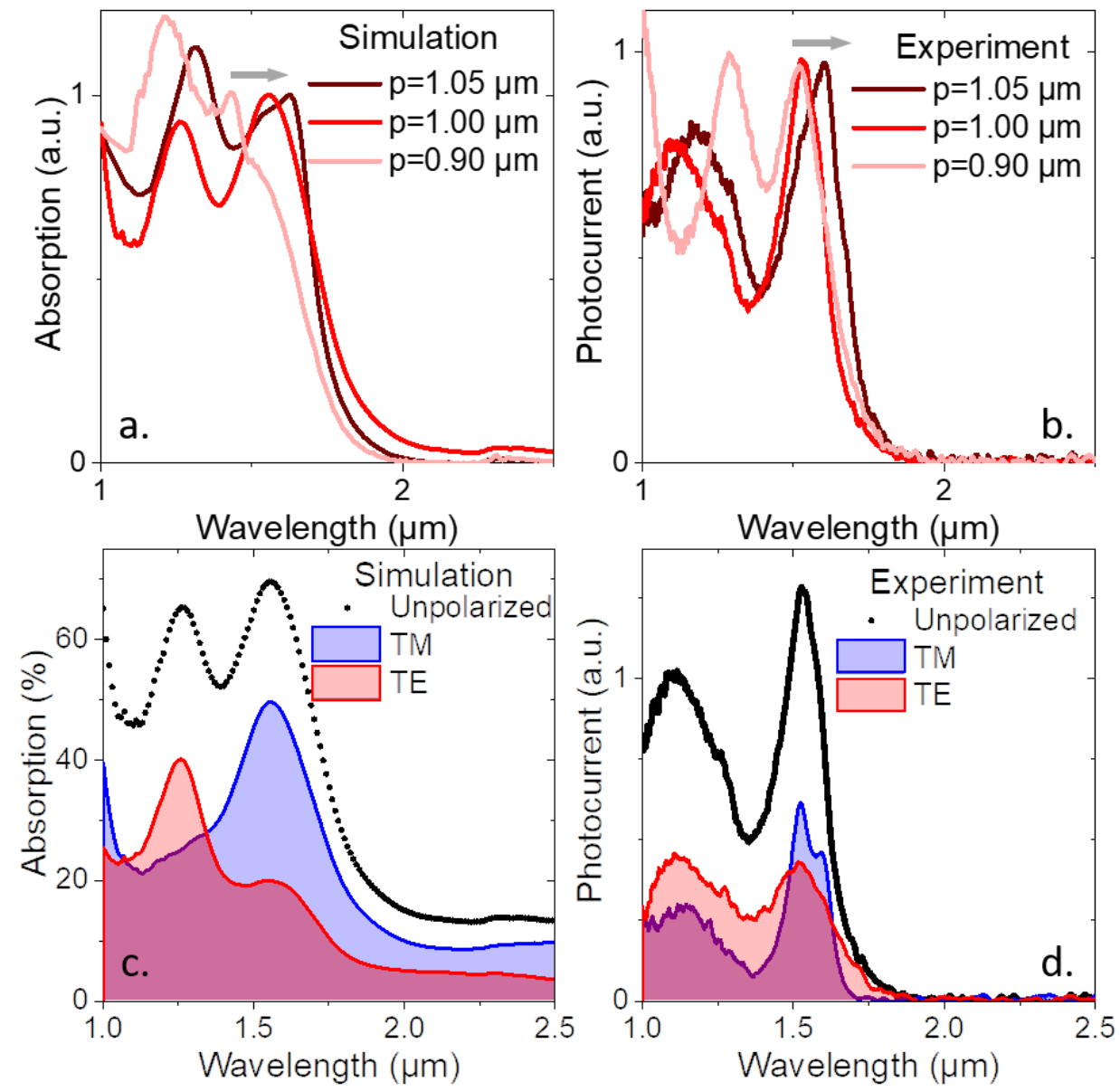

Figure 4 Optical simulations and measurements of GMR structures. a. Simulated absorption and $b$. experimental photocurrent spectra for PbS 6k nanocrystal-based devices with various periods. $c$. Simulated absorption and $d$. experimental photocurrent spectra relative to the total absorption (black dots) and its TM (blue) and TE (red) componants for PbS $6 k$ nanocrystal based devices with a $1 \mu \mathrm{m}$ period.

We have then estimated how the GMR electrodes affect the detection performance of the CQD film. The responsivity of the GMR electrodes reaches 1 A.W'-1 (corresponding to an external quantum efficiency of $86 \%$ ), see Figure $5 \mathrm{a}$. This responsivity is a factor of 250 higher than the one obtained for the same film (same thickness, same applied bias and same operating temperature) using conventional interdigitated electrodes. This increase of the responsivity is also much larger than the increase of the absorption $(\approx 3)$. This clearly brings evidence that the short spacing of the electrodes leads to an increase of the photoconductive gain by a factor of $250 / 3 \approx 80$. Note that the time response of the electrodes remains almost unaffected with a $3 \mathrm{~dB}$ cut-off frequency around $1 \mathrm{kHz}$, see Figure $5 \mathrm{~b}$. The GMR electrodes also leave the nature of the noise unchanged, being still $1 / f$ limited, see Figure $5 \mathrm{c}$. Thanks to the improvement of the responsivity, the detectivity is dramatically enhanced and is close to $10^{9} \mathrm{Jones}$ at room temperature and is above $10^{10}$ Jones at $200 \mathrm{~K}$, see Figure $5 \mathrm{~d}$. Regarding the effect of temperature, it is also worth mentioning that we observe a small redshift of the band 
edge as the sample is cooled down, see Figure S11, but the energies of the resonance peaks remain unaffected. In this sense the GMR electrodes reduce the effect of temperature on the spectra.
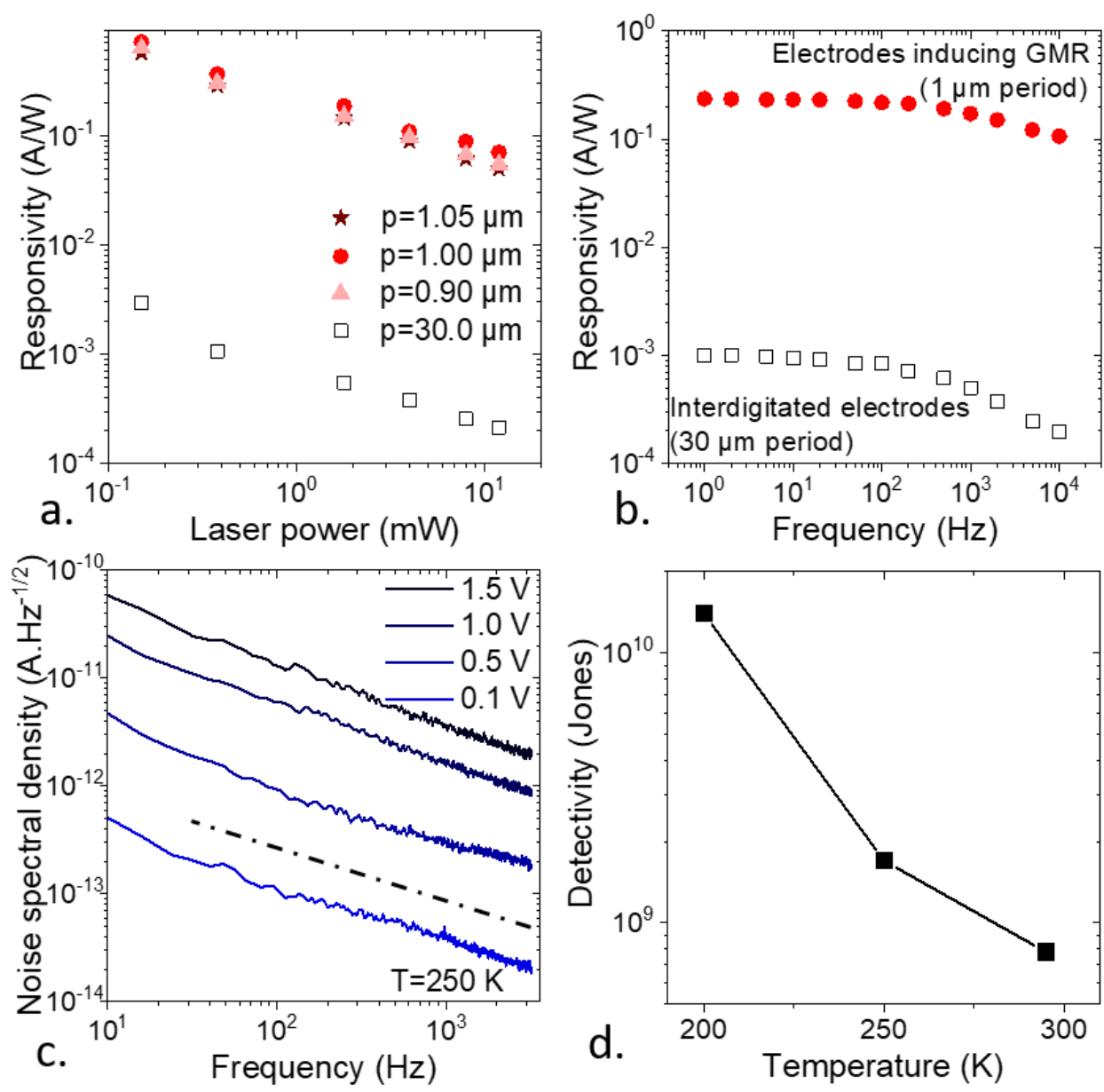

Figure 5 Electrical properties of GMR structures. a. Responsivity as a function of the incident laser beam power $(\lambda=1.55 \mu \mathrm{m})$ for device based on PbS $6 \mathrm{k}$ nanocrystals using interdigitated electrodes made of gold contact deposited on a $\mathrm{Si} \mathrm{SiO}_{2}$ substrate, and interdigitated electrodes on a $\mathrm{Au} / \mathrm{SiO}_{2}$ substrate designed to induce GMR with various period values. In all cases, the applied bias is $1 \mathrm{~V}$. b. Responsivity as a function of the light chopping frequency for device based on PbS 6k nanocrystals using interdigitated electrodes made of gold contact deposited on a $\mathrm{Si} \mathrm{SiO}_{2}$ substrate, and interdigitated electrodes on a $\mathrm{Au} / \mathrm{SiO}_{2}$ substrate designed to induce GMR with $1 \mu \mathrm{m}$ period. c. Noise spectral density as a function of signal frequency for various applied biases at $250 \mathrm{~K}$ measured on the device with interdigitated electrodes on a $\mathrm{Au} / \mathrm{SiO}_{2}$ substrate designed to induce GMR with $1 \mu \mathrm{m}$ period. Dash line corresponds to $1 / \mathrm{f}$ noise decay. $d$. Detectivity as a function of temperature measured on the device with interdigitated electrodes on an $\mathrm{Au} / \mathrm{SiO}_{2}$ substrate designed to induce GMR with $1 \mu \mathrm{m}$ period.

In the last part of the paper we extend the obtained results on PbS in the SWIR range to HgTe nanocrystals in the E-SWIR range $(2.6 \mu \mathrm{m})$, see Figure 6. Because the exciton 
peak is shifted toward longer wavelengths, the period of the GMR is also increased, typically around $1.8 \mu \mathrm{m}$. Figure 6 a shows a comparison of the absorption spectrum for the three types of electrodes proposed in Figure 2, using this time an HgTe CQD layer. Again, we obtain a very good qualitative and quantitative agreement between the simulated and experimental spectra, see Figure $6 \mathrm{c}$ and $\mathrm{d}$.

This time, the enhancement of the absorption is even more dramatic, reaching a factor of $\approx 6$, see Figure $6 \mathrm{a}$. The spectrum of the GMR device becomes driven by the shape of the resonance rather than the shape of the excitonic transition. As a result, the cutoff wavelength can be strongly tuned thanks to the geometrical parameters, see Figure $6 e$ and f. Tuning the period of the GMR electrodes from 1.5 to $1.9 \mu \mathrm{m}$ shifts the peak cut-off wavelength from 2.15 to $2.5 \mu \mathrm{m}$.

As for $\mathrm{PbS}$, the introduction of the GMR electrodes dramatically enhances the responsivity by three orders of magnitude, see Figure $6 \mathrm{~b}$. Values above $1 \mathrm{~A}^{-\mathrm{W}^{-1}}$ are also reached for this material corresponding to an external quantum efficiency of 342 $\%$. Such values above $100 \%$ are again a clear evidence that gain plays a critical role in the improvement of the device performance. 

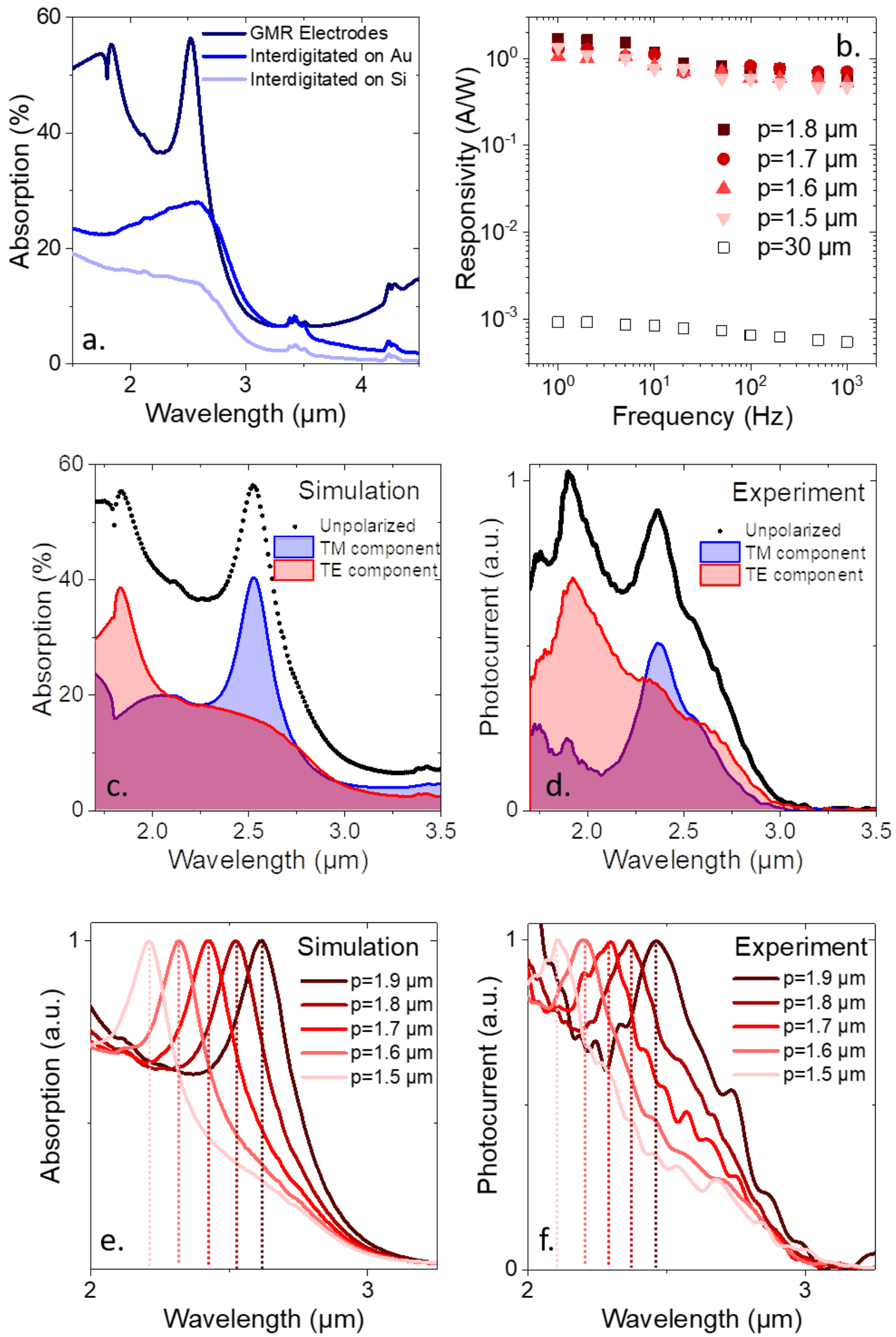
Figure 6: Study of a GMR structure operating at $2.6 \mu \mathrm{m}$ using $\mathrm{HgTe}$ nanocrystals. a. Absorption spectra for device based on $\mathrm{HgTe} 4 \mathrm{k}$ nanocrystals using interdigitated electrodes made of gold contact deposited on a $\mathrm{Si}^{-S_{\mathrm{SO}}}{ }_{2}$ substrate (dark blue), interdigitated electrodes made of gold contact deposited on a $\mathrm{Au} / \mathrm{SiO}_{2}$ substrate (blue) and interdigitated electrodes on a $\mathrm{Au} / \mathrm{SiO}_{2}$ substrate designed to induce GMR (light blue) with a period of $1.8 \mu \mathrm{m}$. b. Responsivity as a function of the light signal frequency for device based on $\mathrm{HgTe} 4 \mathrm{k}$ nanocrystals using interdigitated electrodes made of gold contact deposited on a $\mathrm{Si} / \mathrm{SiO}_{2}$ substrate, and interdigitated electrodes on a $\mathrm{Au} / \mathrm{SiO}_{2}$ substrate designed to induce GMR with various period values. The light source is a blackbody at $980{ }^{\circ} \mathrm{C}$. The incident power is calculated to be $200 \mu \mathrm{W}$ for conventional interdigitated electrodes and $1.7 \mu \mathrm{W}$ for electrode inducing GMR. c. Simulated absorption and $d$. experimental photocurrent spectra relative to TE mode (red), TM mode (blue) and unpolarized (black) absortion for $\mathrm{HgTe} 4 \mathrm{k}$ nanocrystal based device with a $1.8 \mu \mathrm{m}$ period. e. Simulated absorption and $f$. experimental photocurrent spectra for $\mathrm{HgTe} 4 \mathrm{k}$ nanocrystal based device with various periods.

To conclude, we have proposed a new geometry of electrodes for the design of infrared photodetectors based on CQD film in planar geometry. Thanks to the introduction of GMR, near-unity absorption can be achieved at least along one polarization. At the device scale, this corresponds to an increase of the absorption by a factor of 3 to 6 compared to what can be obtained with similar films deposited on conventional interdigitated electrodes. An excellent agreement between simulation and experiment is obtained thanks to a design of electrodes which takes into account the fragility of the CQDs. The enhancement of the responsivity can be as high as a factor of 1000 for $\mathrm{HgTe}$ CQDs. Beyond the increase of the absorption (x 3-6) the electrodes designed for the GMR introduce gain which is typically responsible for a factor of $\approx 100$ in the increase of the response. This strategy is quite versatile and can be applied to several colloidal materials especially in the infrared range. The overall performance of the device becomes fully noise-limited.

\section{Methods}

Chemicals for nanocrystal synthesis. Mercury chloride $\left(\mathrm{HgCl}_{2}\right.$, Strem Chemicals, 99\%), tellurium powder (Te, Sigma-Aldrich, 99.99\%), trioctylphosphine (TOP, Cytek, 90\%), oleylamine (OLA, Acros, 80-90\%), dodecanethiol (DDT, Sigma-Aldrich, 98\%), lead chloride ( $\mathrm{PbCl}_{2}$, Afla Aesar, 99\%), sulfur powder (S, Afla Aesar, 99.5\%), oleic acid (OA, Afla Aesar, 90\%), 1,2,ethanedithiol (EDT, Fluka, 98.0\%), lithium perchlorate $\left(\mathrm{LiClO}_{4}\right.$, Sigma-Aldrich, 98\%), polyethylene glycol (PEG, $\left.\mathrm{Mw}_{\mathrm{w}}=6 \mathrm{~kg} \cdot \mathrm{mol}^{-1}\right)$, chloroform (VWR), ethanol absolute anhydrous (VWR), methanol (Carlo Erba, 99.8\%), acetone (VWR), n-hexane (VWR), toluene (Carlo Erba, 99.3\%), N,N-dimethylformamide (DMF, Sigma Aldrich), mercaptoalcohol (MpOH, Merck, $>99 \%$ ), Methylisobutylketone (MIBK, VWR, $>98.5 \%$ ). All chemicals are used as received, except oleylamine which is 
centrifuged before use. Mercury and lead salts are toxic, handle them with great care.

1 M TOP:Te precursor. In a three-neck flask, $2.54 \mathrm{~g}$ of Te powder is mixed in $20 \mathrm{~mL}$ of TOP. The flask is kept under vacuum at room temperature for $5 \mathrm{~min}$ and then is heated up to $100^{\circ} \mathrm{C}$ and kept under vacuum for 20 more minutes. The atmosphere is changed to $\mathrm{Ar}$ and the temperature is raised to $275^{\circ} \mathrm{C}$. The solution is stirred until a clear orange coloration is obtained. Then the flask is cooled down and the color switches to yellow. The flask is degassed again for $10 \mathrm{~min}$ at room temperature. This solution is finally transferred to an Ar-filled glovebox for storage.

PbS CQD Synthesis. In a three-neck flask, $300 \mathrm{mg}$ of $\mathrm{PbCl}_{2}$ and $7.5 \mathrm{~mL}$ of OLA are degassed, first at room temperature and then at $110^{\circ} \mathrm{C}$ for $30 \mathrm{~min}$. Meanwhile $30 \mathrm{mg}$ of $S$ powder is mixed with $7.5 \mathrm{~mL}$ of OLA until full dissolution and an orange clear solution is obtained. Then under $\mathrm{Ar}$ at $160^{\circ} \mathrm{C}$, this solution of $S$ is quickly added to the flask. After 15 minutes, the reaction is quickly quenched by addition of $1 \mathrm{~mL}$ of oleic acid and $10 \mathrm{~mL}$ of hexane. The nanocrystals are precipitated with ethanol and redispersed in toluene. This washing step is repeated one more time. The solution is then centrifuged to remove the unstable phase. The supernatant is precipitated with methanol and redispersed in toluene. Finally, the PbS CQD solution in toluene is filtered through a $0.2 \mu \mathrm{m}$ PTFE filter.

HgTe CQD Synthesis. In a $100 \mathrm{~mL}$ three-neck flask, $543 \mathrm{mg}$ of $\mathrm{HgCl}_{2}$ and $60 \mathrm{~mL}$ of oleylamine (OLA) are degassed at $110{ }^{\circ} \mathrm{C}$ for an hour. The atmosphere is then switched to $\mathrm{Ar}$ and the solution is cooled down to $80^{\circ} \mathrm{C}$. A solution containing $2 \mathrm{~mL}$ of TOP:Te $(1 \mathrm{M})$ and $10 \mathrm{~mL}$ of OLA is quickly added to the flask after warming it up with a heat gun. After 3 minutes, the reaction is quickly quenched by addition of a mixture of $1 \mathrm{~mL}$ of DDT and $9 \mathrm{~mL}$ of toluene. The nanocrystals are precipitated with ethanol and redispersed in toluene. As a second step of cleaning, the nanocrystals are precipitated with methanol and redispersed in toluene. The pellet is eliminated and the supernatant is washed one more time. Finally, the HgTe CQD solution in toluene is filtered through a $0.2 \mu \mathrm{m}$ PTFE filter.

Infrared spectroscopy is conducted using a Fisher IS50 Fourier transform Infrared spectrometer. To measure CQD absorption, we use the spectrometer in ATR configuration. A drop of CQD solution is dried on the diamond cell. The source is a white light and the detector is a DTGS ATR. Spectra are typically acquired between 10 $000 \mathrm{~cm}^{-1}$ and $2000 \mathrm{~cm}^{-1}$ with a $4 \mathrm{~cm}^{-1}$ resolution and averaging over 32 spectra. To measure a film absorption, an extended InGaAs detector is used and the source is a white light. In case of extended SWIR device, the visible light is switch to a globar source combined with a DTGS detector. Each spectrum is averaged 32 times and the resolution is set to $4 \mathrm{~cm}^{-1}$. Photocurrent spectra are acquired as the sample is biased using a Femto DLPCA 200 current amplifier which role is also to magnify the current. The signal is then fed into the FTIR acquisition board. 
For TEM pictures, a drop of a diluted CQD solution is drop-casted on a copper grid covered with an amorphous carbon film. JEOL $2010 \mathrm{~F}$ is used at $200 \mathrm{kV}$ for acquisition of picture. For device characterization, a FEI Magellan scanning electron microscope is used.

For SEM pictures, a Zeiss Supra 40 scanning electron microscope is used. The acceleration bias is set a $10 \mathrm{kV}$ and the aperture at $20 \mu \mathrm{m}$.

$D C$ Transport. The sample is connected to a Keithley 2634b, which controls the drain bias (VDS) and measures the associated current (IDS). This measure is carried out in the dark or under illumination using $1.55 \mu \mathrm{m}$ laser diode.

Responsivity measurement. For PbS 6k, the source is a laser diode at $1.55 \mu \mathrm{m}$ placed at $20 \mathrm{~cm}$ of the sample. The total spot power is measured to be $0.15 \mathrm{~mW}$. The spot size is $1.6 \mathrm{~mm}^{2}$, corresponding to irradiance of $9.4 \mathrm{~mW} . \mathrm{cm}^{-2}$. The flux is chopped form $1 \mathrm{~Hz}$ to $10 \mathrm{kHz}$. The photocurrent is measured using Zurich Instruments MFLI lock-in amplifier at $1 \mathrm{~V}$ bias. For $\mathrm{HgTe} 4 \mathrm{k}$, the source is a black body at $980{ }^{\circ} \mathrm{C}$ placed at 20 $\mathrm{cm}$ of the sample. A germanium filter is used to suppress the high energy part of the blackbody spectrum. The total power is measured to be $200 \mu \mathrm{W}$ for interdigitated electrodes $\left(2.5 \mathrm{~mm}^{2}\right)$ and $1.7 \mu \mathrm{W}$ for nanoelectrodes. The flux is chopped form $1 \mathrm{~Hz}$ to $1 \mathrm{kHz}$. The photocurrent is measured using Zurich Instruments MFLI lock-in amplifier at $1 \mathrm{~V}$ bias.

Noise measurement. Current from the device (at $1 \mathrm{~V}$ bias, kept in the dark) is amplified by a Femto DLPCA-200, then fed into a SRS SR780 signal analyzer.

Detectivity determination. The detectivity (in Jones) of the sample is determined using the formula: $D^{*}=\frac{R \sqrt{A}}{S_{I}}$, where $\mathrm{R}$ (in $\mathrm{A} \cdot \mathrm{W}^{-1}$ ) is the responsivity, $\mathrm{S}$ is the noise $(\mathrm{A} / \sqrt{\mathrm{Hz}})$ and $A$ the area of the device $\left(\mathrm{cm}^{2}\right)$.

\section{SUPPORTING INFORMATIONS}

Supporting Information is available from ACS website. They include photoconduction characterization of the materials, details relative to fabrication of the GMR electrodes, input used for electromagnetic simulation and discussion about the robustness of the GMR structure. 


\section{ACKNOWLEDEGMENTS}

EL thanks the support ERC starting grant blackQD (grant $\left.n^{\circ} 756225\right)$. We acknowledge the use of clean-room facilities from the "Centrale de Proximite Paris-Centre". This work has been supported by the Region Ile-de-France in the framework of DIM Nano$\mathrm{K}$ (grant dopQD). This work was supported by French state funds managed by the ANR within the Investissements d'Avenir programme under reference ANR-11-IDEX0004-02, and more specifically within the framework of the Cluster of Excellence MATISSE and also by the grant IPER-Nano2, Nanodose, Copin, Frontal, Graskop. JQ thanks Chinese Scholar council for PhD grant while AC thanks l'Agence Innovation Défense for PhD funding.

\section{Conflict of interest}

The authors declare no conflict of interest.

\section{References}

(1) Chen, H.; Liu, H.; Zhang, Z.; Hu, K.; Fang, X. Nanostructured Photodetectors: From Ultraviolet to Terahertz. Advanced Materials 2016, 28, 403-433.

(2) Goubet, N.; Jagtap, A.; Livache, C.; Martinez, B.; Portalès, H.; Xu, X. Z.; Lobo, R. P. S. M.; Dubertret, B.; Lhuillier, E. Terahertz HgTe Nanocrystals: Beyond Confinement. Journal of the American Chemical Society 2018, 140, 5033-5036.

(3) Kroupa, D. M.; Vörös, M.; Brawand, N. P.; McNichols, B. W.; Miller, E. M.; Gu, J.; Nozik, A. J.; Sellinger, A.; Galli, G.; Beard, M. C. Tuning Colloidal Quantum Dot Band Edge Positions through Solution-Phase Surface Chemistry Modification. Nature Communications 2017, 8, 15257.

(4) Koh, W. K.; Koposov, A. Y.; Stewart, J. T.; Pal, B. N.; Robel, I.; Pietryga, J. M.; Klimov, V. I. Heavily Doped N-Type PbSe and PbS Nanocrystals Using GroundState Charge Transfer from Cobaltocene. Scientific reports 2013, 3, 2004.

(5) Robin, A.; Livache, C.; Ithurria, S.; Lacaze, E.; Dubertret, B.; Lhuillier, E. Surface Control of Doping in Self-Doped Nanocrystals. ACS Appl. Mater. Interfaces 2016, 8, 27122-27128.

(6) Brown, P. R.; Kim, D.; Lunt, R. R.; Zhao, N.; Bawendi, M. G.; Grossman, J. C.; Bulović, V. Energy Level Modification in Lead Sulfide Quantum Dot Thin Films through Ligand Exchange. ACS Nano 2014, 8, 5863-5872.

(7) Nag, A.; Kovalenko, M. V.; Lee, J.-S.; Liu, W.; Spokoyny, B.; Talapin, D. V. MetalFree Inorganic Ligands for Colloidal Nanocrystals: $\mathrm{S}^{2-}, \mathrm{HS}^{-}, \mathrm{Se}^{2-}, \mathrm{HSe}^{-}, \mathrm{Te}^{2-}$, $\mathrm{HTe}^{-}, \mathrm{TeS}_{3}{ }^{2-}, \mathrm{OH}^{-}$, and $\mathrm{NH}_{2}{ }^{-}$as Surface Ligands. J. Am. Chem. Soc. 2011, 133, 10612-10620.

(8) Tang, J.; Kemp, K. W.; Hoogland, S.; Jeong, K. S.; Liu, H.; Levina, L.; Furukawa, M.; Wang, X.; Debnath, R.; Cha, D.; et al. Colloidal-Quantum-Dot Photovoltaics Using Atomic-Ligand Passivation. Nature Materials 2011, 10, 765-771.

(9) Choi, J.-H.; Fafarman, A. T.; Oh, S. J.; Ko, D.-K.; Kim, D. K.; Diroll, B. T.; Muramoto, S.; Gillen, J. G.; Murray, C. B.; Kagan, C. R. Bandlike Transport in 
Strongly Coupled and Doped Quantum Dot Solids: A Route to High-Performance Thin-Film Electronics. Nano Lett. 2012, 12, 2631-2638.

(10) Kovalenko, M. V.; Scheele, M.; Talapin, D. V. Colloidal Nanocrystals with Molecular Metal Chalcogenide Surface Ligands. Science 2009, 324, 1417-1420.

(11) Lhuillier, E.; Keuleyan, S.; Zolotavin, P.; Guyot-Sionnest, P. Mid-Infrared $\mathrm{HgTe}_{\mathrm{As}} \mathrm{S}_{3}$ Field Effect Transistors and Photodetectors. Advanced Materials 2013, 25, 137-141.

(12) Moreels, I.; Lambert, K.; Smeets, D.; De Muynck, D.; Nollet, T.; Martins, J. C.; Vanhaecke, F.; Vantomme, A.; Delerue, C.; Allan, G.; et al. Size-Dependent Optical Properties of Colloidal PbS Quantum Dots. ACS Nano 2009, 3, 30233030.

(13) Lhuillier, E.; Keuleyan, S.; Guyot-Sionnest, P. Optical Properties of HgTe Colloidal Quantum Dots. Nanotechnology 2012, 23, 175705.

(14) Kershaw, S. V.; Susha, A. S.; Rogach, A. L. Narrow Bandgap Colloidal Metal Chalcogenide Quantum Dots: Synthetic Methods, Heterostructures, Assemblies, Electronic and Infrared Optical Properties. Chem. Soc. Rev. 2013, 42, 30333087.

(15) Lhuillier, E.; Guyot-Sionnest, P. Recent Progresses in Mid Infrared Nanocrystal Optoelectronics. IEEE J. Selected Topics in Quantum Electronics 2017, 23, 6000208.

(16) Hu, C.; Gassenq, A.; Justo, Y.; Devloo-Casier, K.; Chen, H.; Detavernier, C.; Hens, Z.; Roelkens, G. Air-Stable Short-Wave Infrared PbS Colloidal Quantum Dot Photoconductors Passivated with $\mathrm{Al}_{2} \mathrm{O}_{3}$ Atomic Layer Deposition. Appl. Phys. Lett. 2014, 105, 171110.

(17) Yakunin, S.; Dirin, D. N.; Protesescu, L.; Sytnyk, M.; Tollabimazraehno, S.; Humer, M.; Hackl, F.; Fromherz, T.; Bodnarchuk, M. I.; Kovalenko, M. V.; et al. High Infrared Photoconductivity in Films of Arsenic-Sulfide-Encapsulated LeadSulfide Nanocrystals. ACS Nano 2014, 8, 12883-12894.

(18) Thambidurai, M.; Jang, Y.; Shapiro, A.; Yuan, G.; Xiaonan, H.; Xuechao, Y.; Wang, Q. J.; Lifshitz, E.; Demir, H. V.; Dang, C. High Performance Infrared Photodetectors up to $2.8 \mu \mathrm{m}$ Wavelength Based on Lead Selenide Colloidal Quantum Dots. Opt. Mater. Express 2017, 7, 2326-2335.

(19) Bi, Y.; Pradhan, S.; Gupta, S.; Akgul, M. Z.; Stavrinadis, A.; Konstantatos, G. Infrared Solution-Processed Quantum Dot Solar Cells Reaching External Quantum Efficiency of $80 \%$ at $1.35 \mu \mathrm{m}$ and $\mathrm{J}_{\mathrm{sc}}$ in Excess of $34 \mathrm{~mA} . \mathrm{cm}^{-2}$. Advanced Materials 2018, 30, 1704928.

(20) Killilea, N.; Wu, M.; Sytnyk, M.; Amin, A. A. Y.; Mashkov, O.; Spiecker, E.; Heiss, W. Pushing PbS/Metal-Halide-Perovskite Core/Epitaxial-Ligand-Shell Nanocrystal Photodetectors beyond $3 \mu \mathrm{m}$ Wavelength. Advanced Functional Materials 2019, 29, 1807964.

(21) Kovalenko, M. V.; Kaufmann, E.; Pachinger, D.; Roither, J.; Huber, M.; Stangl, J.; Hesser, G.; Schäffler, F.; Heiss, W. Colloidal HgTe Nanocrystals with Widely Tunable Narrow Band Gap Energies: From Telecommunications to Molecular Vibrations. J. Am. Chem. Soc. 2006, 128, 3516-3517.

(22) Tang, X.; Ackerman, M. M.; Chen, M.; Guyot-Sionnest, P. Dual-Band Infrared Imaging Using Stacked Colloidal Quantum Dot Photodiodes. Nature Photonics 2019, 13, 277.

(23) Jagtap, A.; Martinez, B.; Goubet, N.; Chu, A.; Livache, C.; Gréboval, C.; Ramade, J.; Amelot, D.; Trousset, P.; Triboulin, A.; et al. Design of a Unipolar Barrier for a 
Nanocrystal-Based Short-Wave Infrared Photodiode. ACS Photonics 2018, 5, 4569-4576.

(24) Cryer, M. E.; Halpert, J. E. $300 \mathrm{~nm}$ Spectral Resolution in the Mid-Infrared with Robust, High Responsivity Flexible Colloidal Quantum Dot Devices at Room Temperature. ACS Photonics 2018, 5, 3009-3015.

(25) Chen, M.; Yu, H.; Kershaw, S. V.; Xu, H.; Gupta, S.; Hetsch, F.; Rogach, A. L.; Zhao, N. Fast, Air-Stable Infrared Photodetectors Based on Spray-Deposited Aqueous HgTe Quantum Dots. Advanced Functional Materials 2014, 24, 53-59.

(26) Chen, M.; Lu, H.; Abdelazim, N. M.; Zhu, Y.; Wang, Z.; Ren, W.; Kershaw, S. V.; Rogach, A. L.; Zhao, N. Mercury Telluride Quantum Dot Based Phototransistor Enabling High-Sensitivity Room-Temperature Photodetection at $2000 \mathrm{~nm}$. ACS Nano 2017, 11, 5614-5622.

(27) Tong, J.; Suo, F.; Ma, J.; Tobing, L. Y. M.; Qian, L.; Zhang, D. H. Surface Plasmon Enhanced Infrared Photodetection. Opto-Electron. Adv. 2019, 02, 180026.

(28) Massiot, I.; Vandamme, N.; Bardou, N.; Dupuis, C.; Lemaître, A.; Guillemoles, J.-F.; Collin, S. Metal Nanogrid for Broadband Multiresonant Light-Harvesting in Ultrathin GaAs Layers. ACS Photonics 2014, 1, 878-884.

(29) Massiot, I.; Colin, C.; Péré-Laperne, N.; Roca i Cabarrocas, P.; Sauvan, C.; Lalanne, P.; Pelouard, J.-L.; Collin, S. Nanopatterned Front Contact for Broadband Absorption in Ultra-Thin Amorphous Silicon Solar Cells. Appl. Phys. Lett. 2012, 101, 163901.

(30) Montoya, J. A.; Tian, Z.-B.; Krishna, S.; Padilla, W. J. Ultra-Thin Infrared Metamaterial Detector for Multicolor Imaging Applications. Opt. Express 2017, 25, 23343-23355.

(31) Verdun, M.; Portier, B.; Jaworowicz, K.; Jaeck, J.; Lelarge, F.; Guilet, S.; Dupuis, C.; Haïdar, R.; Pardo, F.; Pelouard, J.-L. Guided-Mode Resonator for Thin InGaAs P-i-N Short-Wave Infrared Photo-Diode. Appl. Phys. Lett. 2016, 108, 053501.

(32) Jackson, E. M.; Nolde, J. A.; Kim, M.; Kim, C. S.; Cleveland, E. R.; Affouda, C. A.; Canedy, C. L.; Vurgaftman, I.; Meyer, J. R.; Aifer, E. H.; et al. TwoDimensional Plasmonic Grating for Increased Quantum Efficiency in Midwave Infrared NBn Detectors with Thin Absorbers. Opt. Express 2018, 26, 1385013864.

(33) Palaferri, D.; Todorov, Y.; Bigioli, A.; Mottaghizadeh, A.; Gacemi, D.; Calabrese, A.; Vasanelli, A.; Li, L.; Davies, A. G.; Linfield, E. H.; et al. Room-Temperature Nine-Mm-Wavelength Photodetectors and GHz-Frequency Heterodyne Receivers. Nature 2018, 556, 85-88.

(34) Yifat, Y.; Ackerman, M.; Guyot-Sionnest, P. Mid-IR Colloidal Quantum Dot Detectors Enhanced by Optical Nano-Antennas. Appl. Phys. Lett. 2017, 110, 041106.

(35) Le-Van, Q.; Le Roux, X.; Aassime, A.; Degiron, A. Electrically Driven Optical Metamaterials. Nature Communications 2016, 7, 12017.

(36) Baek, S.-W.; Ouellette, O.; Jo, J. W.; Choi, J.; Seo, K.-W.; Kim, J.; Sun, B.; Lee, S.-H.; Choi, M.-J.; Nam, D.-H.; et al. Infrared Cavity-Enhanced Colloidal Quantum Dot Photovoltaics Employing Asymmetric Multilayer Electrodes. ACS Energy Lett. 2018, 3, 2908-2913.

(37) Tang, X.; Wu, G. fu; Lai, K. W. C. Plasmon Resonance Enhanced Colloidal HgSe Quantum Dot Filterless Narrowband Photodetectors for Mid-Wave Infrared. J. Mater. Chem. C 2017, 5, 362-369. 
(38) Tang, X.; Ackerman, M. M.; Shen, G.; Guyot-Sionnest, P. Towards Infrared Electronic Eyes: Flexible Colloidal Quantum Dot Photovoltaic Detectors Enhanced by Resonant Cavity. Small 2019, 15, 1804920.

(39) Chen, M.; Shao, L.; Kershaw, S. V.; Yu, H.; Wang, J.; Rogach, A. L.; Zhao, N. Photocurrent Enhancement of HgTe Quantum Dot Photodiodes by Plasmonic Gold Nanorod Structures. ACS Nano 2014, 8, 8208-8216.

(40) Tang, X.; Ackerman, M. M.; Guyot-Sionnest, P. Thermal Imaging with Plasmon Resonance Enhanced HgTe Colloidal Quantum Dot Photovoltaic Devices. ACS Nano 2018, 12, 7362-7370.

(41) Prins, F.; Kim, D. K.; Cui, J.; De Leo, E.; Spiegel, L. L.; McPeak, K. M.; Norris, D. J. Direct Patterning of Colloidal Quantum-Dot Thin Films for Enhanced and Spectrally Selective Out-Coupling of Emission. Nano Lett. 2017, 17, 1319-1325.

(42) De Leo, E.; Cocina, A.; Tiwari, P.; Poulikakos, L. V.; Marqués-Gallego, P.; le Feber, B.; Norris, D. J.; Prins, F. Polarization Multiplexing of Fluorescent Emission Using Multiresonant Plasmonic Antennas. ACS Nano 2017, 11, 12167-12173.

(43) Baek, S.-W.; Molet, P.; Choi, M.-J.; Biondi, M.; Ouellette, O.; Fan, J.; Hoogland, S.; Arquer, F. P. G. de; Mihi, A.; Sargent, E. H. Nanostructured Back Reflectors for Efficient Colloidal Quantum-Dot Infrared Optoelectronics. Advanced Materials 2019, 31, 1901745.

(44) Sharon, A.; Rosenblatt, D.; Friesem, A. A. Resonant Grating-Waveguide Structures for Visible and near-Infrared Radiation. Journal of the Optical Society of America A 1997, 14, 2985.

(45) Keuleyan, S.; Lhuillier, E.; Guyot-Sionnest, P. Synthesis of Colloidal HgTe Quantum Dots for Narrow Mid-IR Emission and Detection. J. Am. Chem. Soc. 2011, 133, 16422-16424.

(46) Martinez, B.; Ramade, J.; Livache, C.; Goubet, N.; Chu, A.; Gréboval, C.; Qu, J.; Watkins, W. L.; Becerra, L.; Dandeu, E.; et al. HgTe Nanocrystal Inks for Extended Short-Wave Infrared Detection. Advanced Optical Materials 2019, 7, 1900348.

(47) Lai, Y.; Li, H.; Kim, D. K.; Diroll, B. T.; Murray, C. B.; Kagan, C. R. Low-Frequency 1/f Noise in Nanocrystal Field-Effect Transistors. ACS Nano 2014, 8, 9664-9672.

(48) Liu, H.; Lhuillier, E.; Guyot-Sionnest, P. 1/f Noise in Semiconductor and Metal Nanocrystal Solids. Journal of Applied Physics 2014, 115, 154309.

(49) De lacovo, A.; Venettacci, C.; Colace, L.; Scopa, L.; Foglia, S. Noise Performance of PbS Colloidal Quantum Dot Photodetectors. Appl. Phys. Lett. 2017, 111, 211104.

(50) Wang, H.; Lhuillier, E.; Yu, Q.; Zimmers, A.; Dubertret, B.; Ulysse, C.; Aubin, H. Transport in a Single Self-Doped Nanocrystal. ACS Nano 2017, 11, 1222-1229.

(51) Konstantatos, G.; Sargent, E. H. PbS Colloidal Quantum Dot Photoconductive Photodetectors: Transport, Traps, and Gain. Appl. Phys. Lett. 2007, 91, 173505.

(52) Lhuillier, E.; Dayen, J.-F.; Thomas, D. O.; Robin, A.; Doudin, B.; Dubertret, B. Nanoplatelets Bridging a Nanotrench: A New Architecture for Photodetectors with Increased Sensitivity. Nano Lett. 2015, 15, 1736-1742.

(53) Chu, A.; Ferre, S.; Noguier, V.; Gréboval, C.; Livache, C.; Martinez, B.; Prado, Y.; Goubet, N.; Cruguel, H.; DuDy, L.; et al. HgTe Nanocrystals for SWIR Detection and Their Integration up to Focal Plane Array. ACS Appl. Mater. Interfaces 2019, asap. 
(54) Bierret, A.; Vincent, G.; Jaeck, J.; Pelouard, J.-L.; Haïdar, R.; Pardo, F. Field Extension inside Guided-Mode-Resonance Filters under a Focused Beam. Opt. Lett. 2017, 42, 4187-4190.

(55) Kondo, T.; Ura, S.; Magnusson, R. Design of Guided-Mode Resonance Mirrors for Short Laser Cavities. J. Opt. Soc. Am. A 2015, 32, 1454-1458.

(56) Bierret, A.; Vincent, G.; Jaeck, J.; Pelouard, J.-L.; Pardo, F.; Barrière, F. D. L.; Haïdar, R. Pixel-Sized Infrared Filters for a Multispectral Focal Plane Array. Appl. Opt. 2018, 57, 391-395.

(57) Hugonin, J.-P.; Lalanne, P. Reticolo Software for Grating Analysis. Reticolo software for grating analysis, Institut d'Optique, Orsay, France 2005. 
TOC graphic

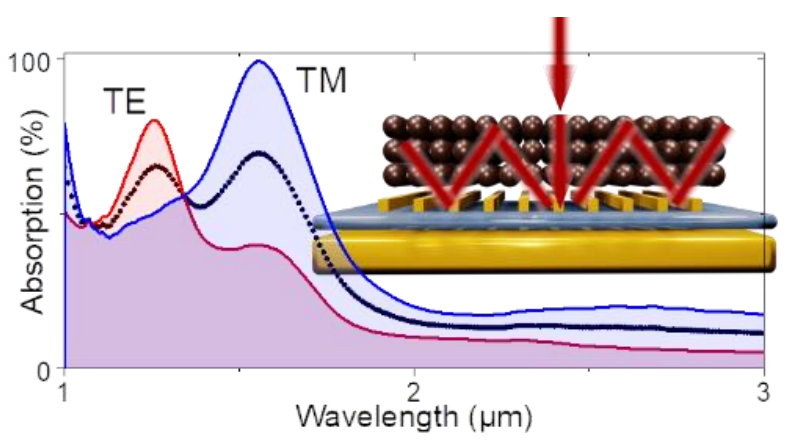

